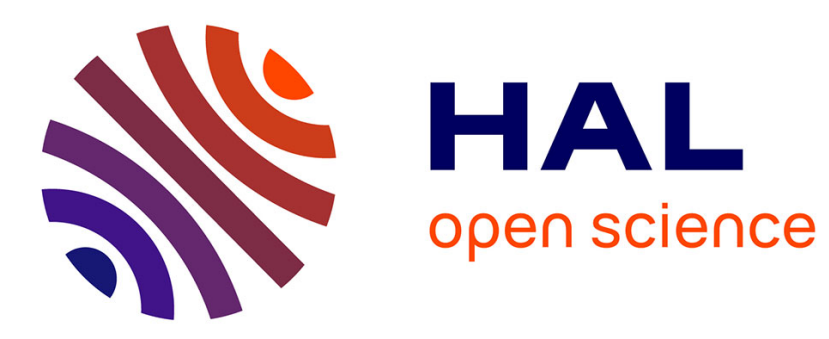

\title{
Optimizing the trajectory of drones: trade-off between distance and energy
}

Igor Dias da Silva, Christelle Caillouet

\section{To cite this version:}

Igor Dias da Silva, Christelle Caillouet. Optimizing the trajectory of drones: trade-off between distance and energy. IAUV 2020 - 2nd International Workshop on Internet of Autonomous Unmanned Vehicles, Jun 2020, Cuomo, Italy. 10.1109/SECONWorkshops50264.2020.9149781 . hal-02779495

\section{HAL Id: hal-02779495 \\ https://hal.inria.fr/hal-02779495}

Submitted on 4 Jun 2020

HAL is a multi-disciplinary open access archive for the deposit and dissemination of scientific research documents, whether they are published or not. The documents may come from teaching and research institutions in France or abroad, or from public or private research centers.
L'archive ouverte pluridisciplinaire HAL, est destinée au dépôt et à la diffusion de documents scientifiques de niveau recherche, publiés ou non, émanant des établissements d'enseignement et de recherche français ou étrangers, des laboratoires publics ou privés. 


\section{Optimizing the trajectory of drones: trade-off between distance and energy}

\author{
Igor Dias Da Silva \\ Centro de Informática (CIn) \\ Universidade Federal de Pernambuco \\ Recife-PE, Brazil \\ ids3@cin.ufpe.br
}

\author{
Christelle Caillouet \\ Université Côte d'Azur, CNRS, I3S, Inria \\ Sophia Antipolis, France \\ christelle.caillouet@univ-cotedazur.fr
}

\begin{abstract}
The use of autonomous unmanned aerial vehicles (UAVs) or drones has emerged to efficiently collect data from mobile sensors when there is no infrastructure available. The drones can form a flying ad-hoc network through which the sensors can send their data to a base station at any time. In this paper, we present a mixed integer linear program to find the drones' optimal trajectories to form and maintain this network through time while minimizing their movements and energy consumption. Furthermore we analyze the trade-off between distance and energy, where increasing the drones' mobility can reduce their energy consumption, and derive a fair trade-off optimal solution to balance the two opposite objectives.
\end{abstract}

\section{INTRODUCTION}

This paper address the problem of aerial data collection in which we use drones to collect data from a set of mobile sensors located on the ground. Data collection with drones has several applications for environmental monitoring or surveillance such as: wildlife monitoring [1], agricultural environment [2], disaster assistance operations [3], and vehicle observation and tracking [4].

In a disaster scenario, the more information we can collect, the more efficiently we can manage the situation, and therefore, more lives can be saved. For this, mobile sensors can be deployed to scan the environment and obtain critical information. For example, the mobile sensors can help us identify the location of victims that need to be rescued. Such critical information must be collected as fast as possible. But in these extreme scenarios, we cannot assume a functional preexisting networking infrastructure that the mobile sensors could use to send their data.

In agricultural or wildlife monitoring, the nodes are moving in smart farming environments to capture specific behaviors related to the quality of crops and the well-being of animals. The data collection in these cases is a difficult task due to the lack of reliable and constant connection with the base station. Moreover, the network is usually deployed in rural areas where the cellular coverage is poor.

To fill the lack of networking infrastructure, the use of drones has emerged as an effective low cost solution [5]. To collect the data from the mobile sensors efficiently, the drones can form a Flying Ad-Hoc Network (FANET) [6] to form the infrastructure through which the mobile sensors can send their information to a fixed base station.

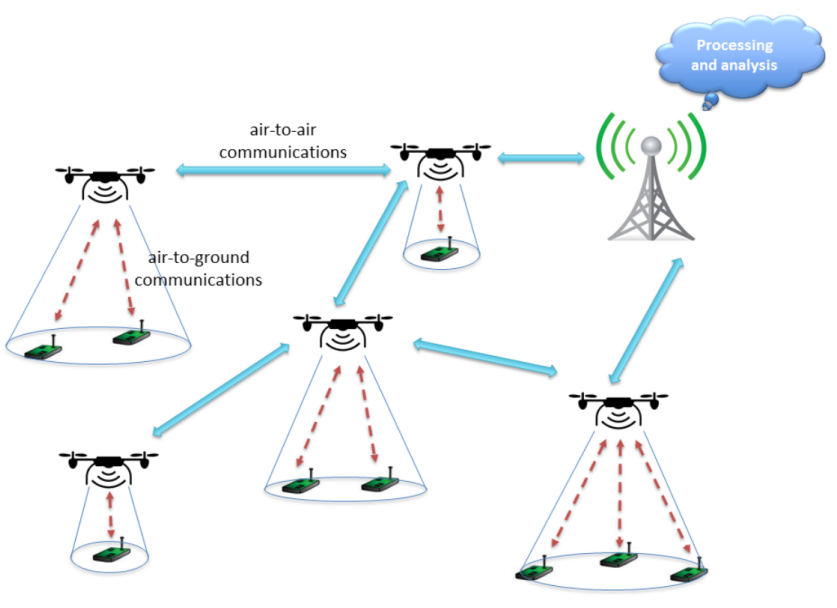

Fig. 1: The network architecture [7].

It is important to build this network such that every mobile sensor is connected to it while providing a backbone to the base station at all times. If we can provide a network satisfying these constraints, then the mobile sensors are connected to the base station at all times and can send the data as soon as it is produced. An example of such a network architecture is shown in Figure 1.

However, to efficiently deploy the drones to collect the data, we not only want to connect all mobile sensors to the base station at all times but also to manage efficiently the FANET. Several parameters are important and must be optimally chosen while deploying this type of network. First, the number of available drones is important because the FANET must be connected so the drones can be used either for coverage and/or connectivity. We also want to minimize parameters such as the drones' altitude to improve the quality of communication. And to guarantee long term connectivity, the lifetime of the network must be maximized. This latter objective must be ensured by properly managing the energy consumption and the total traveled distance of the drones.

In this paper we provide mixed integer linear programs that build the Flying Ad-Hoc Network such that the mobile sensors are connected to the base station at all times and minimize the total traveled distance by the drones and the total consumed 
energy.

The rest of the paper is organized as follows. We overview the existing work about optimal FANET deployment and the different energy consumption models in Section II. Then, we develop our optimization models with the objectives of minimizing the total traveled distance of the drones and their energy consumed while traveling in Section III. Section IV presents the results obtained on the test instances and the trade-off analysis between distance and energy to derive a fair optimal solution optimizing both objectives. Finally, Section V concludes the work and derives the following perspectives.

\section{RELATED WORK}

To cover a set of targets, D. Zorbas et al [8] looked for the optimal deployment of the drones such that each target is covered by at least one drone while minimizing the energy cost and the number of deployed drones. Continuing this work, the same authors proposed a new model that considers that the energy of each drone is not enough for monitoring the targets for the entire observation period [9]. Therefore the drones must be replaced when they run out of battery. This is done by adding non-linear constraints in the mathematical formulation.

Zhen et al [10] consider the problem of FANET deployment as a vehicle routing problem (VRP), where one must determine not only the order in which to visit a set of ground nodes but also the altitude at which to visit them. Authors present an integer linear program and a tabu search metaheuristic to optimize flight routes and minimize the total time needed to complete the monitoring tasks.

For all these works the energy consumption is assumed to be related to the drone's altitude. The higher the drone is, the more energy it consumes, but at the same time, the bigger is its coverage radius. The authors presume according to their measurements and the drone manufacturers' specifications that this model is not far from reality.

The works presented above provide the trajectories for the drones but they do not account for the connectivity constraint we are interested in. We want to provide a connected path from every mobile sensor to the base station at all times. Considering this, authors of [11] provide a mixed integer linear program to optimally select the drone locations and altitude to cover fixed targets and form a connected FANET. They also provided a trade-off analysis between the number of drones and their altitude to balance between cost and reliability of communications.

C. Caillouet et al [7] then extended the formulation to find the optimal set of positions where the drones should be deployed to cover all mobile targets during an observation period while maintaining a backbone to a base station. The model presented minimizes the number of deployed drones, their altitude to provide better communication quality and it minimizes the flying distance from the base station to reduce the energy consumption that was assumed to be directly related to the traveled distance. Although this latter model takes into consideration all the constraints we are interested in, it does not associate the drones with the positions so we cannot know specifically the trajectory of each drone.

Regarding the energy consumption of drones, some works in the literature such as [12] and [13] provide more detailed models in aspect to the theoretical understanding of flights. In [12] the energy consumption is directly related to the drone's flying speed, weight and payload, and air density.

In [13], the author considers the communication related power to be constant and the propulsion power consumption consisting of three components: blade profile, induced, and parasite power. These components are detailed derived considering the air density, the drone's weight, its rotor radius, blade angular velocity, and many other physical characteristics.

The two models show that the energy consumption of the drone does not behave linearly with the speed or traveled distance. For some speeds the energy consumption is actually lower than if the drone was simply hovering a position. This means that reducing the traveled distance does not necessarily reduce the energy consumed and this was not considered in [7], [8], and [9].

To minimize the energy consumption of the drones, we apply the energy model of [13] into our linear integer program because it is much more precise than what is considered in [8], [9], and [7]. And the paper provides all the information we needed to implement the same model in our program.

\section{OPTIMIZATION MODELS}

As in [7], we consider a discrete observation period $t \in$ $[0, T]$. We first define the set of the ground sensors positions in time step $t$ as $N^{t}$. So a ground sensor $s$ at time step $t$ has a position $\left(x_{s}, y_{s}\right)$ such that $\left(x_{s}, y_{s}\right) \in N^{t}$. We use $b$ to define the base station whose coordinates are $(0,0,0)$ and $P$ to represent the set of deployment positions in the $3 D$ space where the drones can be placed.

In these positions we can deploy up to $M$ drones. For a drone $m$ placed in a position $p=\left(x_{p}, y_{p}, h_{p}\right) \in P$, we define its communication range as $R_{p}$ and its coverage radius as $r_{p}$ :

$$
r_{p} \leq h_{p} \cdot \tan \frac{\theta}{2}
$$

The higher the drone is, the bigger its coverage radius is. We consider that a drone $m$ placed in $p$ covers a sensor $n$ if the distance between $n$ and the projection of $p$ in the $2 D$ plane is smaller than the drone's coverage radius

$$
d(p, n)=\sqrt{\left(x_{p}-x_{n}\right)^{2}+\left(y_{p}-y_{n}\right)^{2}} \leq r_{p}
$$

and it communicates with another drone $v$ if the distance between them is smaller than its communication range.

$$
D_{p, q}=\sqrt{\left(x_{p}-x_{q}\right)^{2}+\left(y_{p}-y_{q}\right)^{2}\left(h_{p}-h_{q}\right)^{2}} \leq R_{p}
$$

We can derive a dynamic graph representation for our aerial data collection problem. For each time step $t$ of the observation period, we define $G^{t}=\left(V^{t}, E^{t}\right)$ where:

$$
V^{t}=\{b\} \cup P \cup N^{t}
$$




$$
\begin{aligned}
E^{t}= & \left\{(b, p), p \in P \text { and }\left(x_{p}, y_{p}\right)=\min _{q \in P} d(b, q)\right\} \cup \\
& \left\{(p, q), p, q \in P \text { and } R_{p} \geq D_{p, q}\right\} \cup \\
& \left\{(p, n), p \in P, n \in N^{t} \text { and } r_{p} \geq d(p, n)\right\}
\end{aligned}
$$

The vertices are the base station $b$, the possible positions to deploy the drones $P$, and the ground sensors' positions at $t$ $N^{t}$. As for the edges set, we say that there is an edge between a sensor and a position if when a drone is deployed in that position, it covers that sensor. And there is an edge between two positions if they are within the communication range. We also consider one edge from the base station to the closest position to ensure global connectivity.

The goal is to determine a set of paths in $G^{t}$ between the base station and the sensors of $N^{t}$, such that there is a drone deployed on each vertex of the paths. Then, the overall goal is to associate one position at each time step $t$ with a drone, such that we minimize the total traveled distance and the energy consumed through time.

\section{A. Optimal formulation}

In each time step we want to determine the position of each drone so we define the binary variables $Z_{p, m}^{t}$ that is 1 if drone $m$ is in position $p$ at time $t$ and it is 0 otherwise. The binary variables $Z_{p, q, m}^{t-1, t}$ on the other hand are 1 if a drone $m$ was in position $p$ at time $t-1$ and moved to position $q$ at time $t$ and are 0 otherwise. We use constraints 1,2 and 3 to ensure that if $Z_{p, m}^{t-1}=1$ and $Z_{q, m}^{t}=1$ then $Z_{p, q, m}^{t-1, t}=1$ and in any other case $Z_{p, q, m}^{t-1, t}=0$.

$$
\begin{gathered}
Z_{p, q, m}^{t-1, t} \leq Z_{p, m}^{t-1} \\
Z_{p, q, m}^{t-1, t} \leq Z_{q, m}^{t} \\
Z_{p, q, m}^{t-1, t} \geq Z_{p, m}^{t-1}+Z_{q, m}^{t}-1 \\
\forall t \in[1, T], \forall p, q \in P \cup\{b\} \text { and } \forall m \in[0, M] \\
Z_{p, m}^{t-1}, Z_{q, m}^{t}, Z_{p, q, m}^{t-1, t} \in[0,1]
\end{gathered}
$$

The set of constraints 4 ensures the definition of the variable $Z_{p}^{t}$. This variable represents how many drones are at position $p$ at time $t . Z_{p}^{t}$ is an integer variable that can take value 0 or $1 \forall p \in P$ and between 0 and $M$ for the position of the base station $b$. Constraints 4 thus ensure that we can not have more than one drone in a position at the same time except for the base station that can hold all $M$ drones.

$$
\begin{gathered}
Z_{p}^{t}=\sum_{m=1}^{M} Z_{p, m}^{t} \quad, \forall t \in[0, T] \text { and } \forall p \in P \cup\{b\} \\
Z_{p}^{t} \in[0, M] \quad \text { if } \mathrm{p}=\mathrm{b} \\
Z_{p}^{t} \in[0,1] \quad \text { if } \mathrm{p} \neq \mathrm{b}
\end{gathered}
$$

We also need to avoid placing the same drone in multiple positions at the same time and for this we use constraints 5 .

$$
\sum_{p \in P \cup\{b\}} Z_{p, m}^{t}=1 \quad, \forall t \in[0, T] \text { and } \forall m \in[0, M]
$$

But when we place the drones we want to ensure that all the mobile sensors have a connection with the base station at all times. In our dynamic graph we can do this by ensuring the existence of a flow from the base station to each mobile sensor in each time step. The flow from vertex $v$ to vertex $u$ at time $t$ is defined by the real variable $f_{v u}^{t}$.

$$
\begin{gathered}
\sum_{l \in V^{t}, l \neq p} f_{p l}^{t}-\sum_{l \in V^{t}, l \neq p} f_{l p}^{t}=\left\{\begin{array}{cl}
\left|N^{t}\right| & \text { if } p=b \\
0 & \text { if } p \in P \\
-1 & \text { if } p \in N^{t}
\end{array}\right. \\
\forall t \in[0, T], p \in V^{t} \\
f_{p l}^{t} \in \mathbb{R}
\end{gathered}
$$

And there can only be a flow between two positions when there are drones placed in both positions which we can ensure with constraints 7 .

$$
f_{p l}^{t} \leq Z_{p}^{t} \cdot\left|N^{t}\right|, \forall t \in[0, T],(p, l) \in E^{t} \text { with } p \in P
$$

All feasible solutions to this model (i.e. solutions fulfilling the above constraints) associate to each drone a position at each time step of the monitored period. The position can be either the base station, in this case the drone is assumed to be unused, or a position $p \in P$, in which case the drone is deployed in the FANET. These successive positions represent the trajectory of the drone through time. All the computed trajectories ensure that the drones cover all mobile sensors and provide a connected path to the base station in all time steps. Now we need to define the objective of the model, in other words the optimal solutions regarding the total traveled distance and the energy consumed by the drones.

\section{B. Model objectives}

1) Distance-based trajectories: To compute the total traveled distance by the drones we use the distance between two positions $D_{p, q}$ defined at the beginning of Section III. From this we define the objective function 8 . The first term of this objective function accounts for the traveled distance in the observation period $[0, T]$. But the drones are initially located at the base station before the observation period, and they must return to the base station when it is over. So the other two terms of the objective function account for the deployment cost before the observation period and the return cost after it, respectively. Note also that if a drone needs to return to the base station during the period, the flying back distance is accounted in the first sum of the objective function, so that the model does not favor changing the drones if not required.

$$
\begin{gathered}
\min \sum_{t=1}^{T} \sum_{p, q \in P \cup\{b\}} \sum_{m=1}^{M} D_{p, q} \cdot Z_{p, q, m}^{t-1, t} \\
+\sum_{p \in P} \sum_{m=1}^{M} D_{p, b} \cdot Z_{p, m}^{0}+\sum_{p \in P} \sum_{m=1}^{M} D_{p, b} \cdot Z_{p, m}^{T}
\end{gathered}
$$

Therefore by minimizing this objective function while satisfying the constraints defined we can obtain the drones' trajectory such that they travel the least. 
2) Energy-based trajectories: To compute the total energy consumed by the drones we adopted the model presented by Y. Zeng et al [13] where the power consumption $P(v)$ of a drone depends on its speed as shown in Figure 2. Here the additional power consumption caused by acceleration and deceleration is ignored. This model considers the blade profile, parasite, and induced powers. The blade profile power and parasite power are needed to overcome the profile drag of the blades and the fuselage drag, respectively. And the induced power is the one required to overcome the induced drag of the blades.

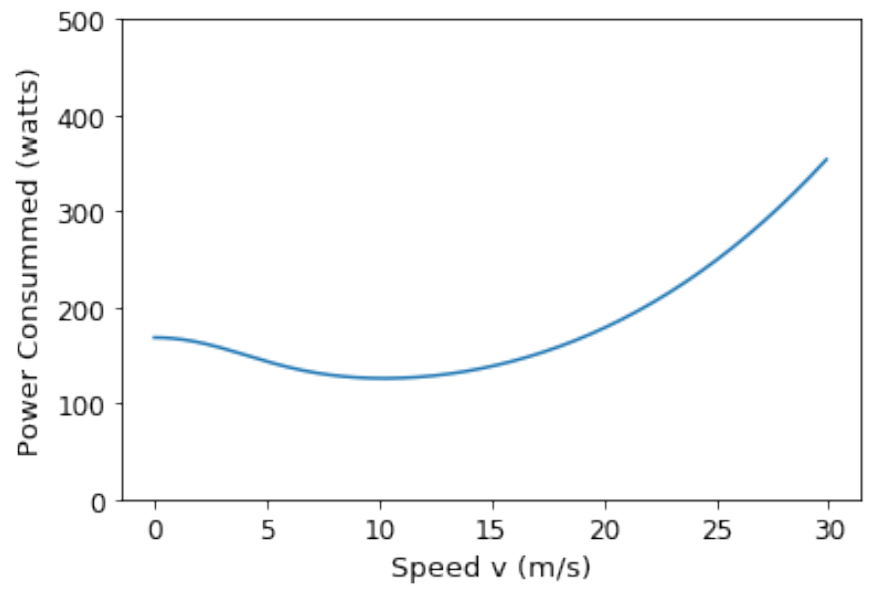

Fig. 2: Power consumed by the drone according to its speed.

To use this model we first need to choose the appropriate travel speed of the drone before computing how much energy it is going to consume. To decide the speed at which the drone travels between positions $p$ and $q$, we first compute the minimal speed, $V_{\min }$, at which it can travel. Because if the drone has to travel 30 meters and only has 1 second between time steps, then it should move at least at $30 \mathrm{~m} / \mathrm{s}$ otherwise the drone will not be in position at the next time step. So it is important to define the time between time steps, $t t s$, that is the time the drones have to travel between each time step and derive $V_{\text {min }}=D_{p, q} /$ tts.

Since we know the drone consumes the minimal amount of power possible at $V_{\text {opt }}=10.2 \mathrm{~m} / \mathrm{s}$, if $V_{\min }$ is greater than $10.2 \mathrm{~m} / \mathrm{s}$, it means $V_{\min }$ is the best speed at which the drone can travel because any faster implies into consuming more energy. But if $V_{\min }$ is less than $10.2 \mathrm{~m} / \mathrm{s}$ it means the drone can go at $10.2 \mathrm{~m} / \mathrm{s}$ arriving before the next time step and hover over the next position for the residual time, this way consuming less energy. So we define that the drone travels with speed $v=\max \left(V_{\min }, V_{\text {opt }}\right)$.

Then we can compute the energy consumed by a drone when traveling between two positions at a speed $v$ that is the energy consumed by the travel plus the energy consumed in case the drone arrives early and it needs to hover the position for the remaining time until the next time step:

$$
E_{p, q}=P(v) \frac{D_{p, q}}{v}+E_{\text {hovering }}
$$

$$
E_{\text {hovering }}=\left\{\begin{array}{cl}
P(0)\left(t t s-\frac{D_{p, q}}{v}\right) & \text { if } p \text { and } q \in P \\
0 & \text { otherwise }
\end{array}\right.
$$

We only consider the energy of hovering when both positions $p$ and $q$ are in $P$ because if the drone is going from a position in $P$ to the base station and it goes faster than $V_{\text {min }}$, it doesn't need to hover the base station until the next time step waiting to land, it can land immediately. And if the drone is coming from the base station to a position in $P$ and it can travel faster than $V_{\min }$, than it should delay its departure from the base station to avoid hovering the position unnecessarily.

Finally, the objective function that computes the total energy consumed by the drones is defined as:

$$
\begin{gathered}
\min \sum_{t=1}^{T} \sum_{p, q \in P \cup\{b\}} \sum_{m=1}^{M} E_{p, q} \cdot Z_{p, q, m}^{t-1, t} \\
+\sum_{p \in P} \sum_{m=1}^{M} E_{p, b} \cdot Z_{p, m}^{0}+\sum_{p \in P} \sum_{m=1}^{M} E_{p, b} \cdot Z_{p, m}^{T}
\end{gathered}
$$

3) Distance-Energy trade-off: Here we define the total traveled distance $D_{\text {total }}$ as the sum of the members of equation 8 and the total energy consumed $E_{\text {total }}$ as the sum in equation 9. The combined objective function to study the trade-off between distance and energy is set as:

$$
\min (1-\alpha) D_{\text {total }}+\alpha \beta E_{\text {total }}
$$

The input parameter $\alpha \in[0,1]$ allows us to choose how much weight we want to give to either the distance or the energy when minimizing the objective function 10 . On the other hand the constant $\beta=V_{\text {opt }} / P_{\min }$ is used to reduce the energy consumed measured in Joules to a magnitude comparable to meters, therefore, avoiding $E_{\text {total }}$ to be the most weighted term in the objective function. With this combined objective we are now able to study carefully the trade-off between the two optimizations.

\section{RESUlTS}

\section{A. Scenarios}

The models presented were implemented using the Java API of IBM Cplex solver 12.10.0 in a computer with an Intel(R) Core(TM) i7-7700HQ CPU $2.80 \mathrm{GHz}, 8 \mathrm{~Gb}$ RAM, running Ubuntu 18.04.4 LTS operating system. The memory required to find the optimal solutions can be very high so we set an $80 \mathrm{~Gb}$ swap file.

As for the default parameter values in our experiments we have: the number of deployment positions varies between 9 , 16 and 25 positions that are distributed as a grid in the $100 \mathrm{~m}^{2}$ square at a height of $45 \mathrm{~m}$. We use $M=5$ drones with a $60 \mathrm{~m}$ communication range to cover 5 mobile sensors. The period of observation was 7 time steps with the default time between time steps set as 2 seconds. The sensors move according to the random waypoint model and we vary their speeds between 5 and $20 \mathrm{~m} / \mathrm{s}$. 
These parameter values were chosen because we can obtain optimal solutions deriving theoretical bounds on the drones' deployment and trajectory characteristics. But even these small scenarios can require a lot of time and memory to find the optimal solution. The number of deployment positions changes the size of the dynamic graph built and therefore impacts the number of variables and constraints of the mixed integer linear program. The same can be said for the number of time steps. The positions height also have a big impact, higher heights give us a bigger coverage radius for the drones. If the drones have a bigger coverage radius we need fewer drones to solve the problem and they can move less. We have also chosen a big communication range to reduce the number of drones necessary to build the path from the base station to the mobile sensors.

The objective here is to present optimal solutions for small scenarios, allowing us to highlight drone behavior when we choose to optimize its total flying time or its total energy consumed. An analysis of the different behaviors is presented in the next subsection, before proposing an effective trade-off solution to make these two objectives be jointly minimized.

\section{B. Results with one objective}

We computed the trajectories that minimize the total traveled distance for the 9 traces of the mobile sensors using 9, 16 and 25 deployment positions each, totaling 27 experiments. The average total traveled distance per experiment when there were 9 deployment positions was $642 m$ while the average with 25 deployment positions was $568 \mathrm{~m}$. This happens because the more positions available, the more precise is the movement of the drones so unnecessary movements are not made. We can verify it when we look at the total distance traveled during the observation period, which is the total traveled distance minus the distance traveled on the deployment and return phases, shown as the partial distance in Table I. All results on this table were obtained with the objective function 8 .

TABLE I: Results when minimizing traveled distance.

\begin{tabular}{|c|c|c|c|c|}
\hline$|P|$ & Total dst & Partial dst & Energy & Time \\
\hline 9 & $642.62 \mathrm{~m}$ & $41.78 \mathrm{~m}$ & $36389.42 \mathrm{~J}$ & $3.5 \mathrm{sec}$ \\
16 & $645.24 \mathrm{~m}$ & $21.85 \mathrm{~m}$ & $47050.98 \mathrm{~J}$ & $5.46 \mathrm{~min}$ \\
25 & $568.20 \mathrm{~m}$ & $20.03 \mathrm{~m}$ & $48514.66 \mathrm{~J}$ & $2.82 \mathrm{~h}$ \\
\hline
\end{tabular}

TABLE II: Results when minimizing consumed energy.

\begin{tabular}{|c|c|c|c|c|}
\hline$|P|$ & Total dst & Partial dst & Energy & Time \\
\hline 9 & $956.03 \mathrm{~m}$ & $443.19 \mathrm{~m}$ & $16558.69 \mathrm{~J}$ & $12.6 \mathrm{~min}$ \\
\hline
\end{tabular}

Although increasing the number of positions leads to more precise movements, it also has a big impact on the resolution time since it increases the number of variables and constraints. Table I shows the average resolution time to find the optimal solution that increases very quickly with the number of positions.

When minimizing the energy consumption we only used 9 deployment positions along with all the default parameter values. As shown in Table II, with the objective function 9, the average traveled distance by the drones during the observation period goes to $443.19 \mathrm{~m}$ which is 10 times higher than when we minimize the total traveled distance but the average energy consumed is reduced to 16558.69 Joules, which is at least 2 times lower than in the distance optimization.

We can see that the drones will often exchange positions when close to each other to avoid hovering the same position. This way they consume less energy while increasing the total traveled distance. In Figure 3 for example, we have the drones' positions at the time steps 4 and 5 of the optimal solution found for an experiment. The blue edges show that the drones are within communication range and the red circles show the coverage area of each drone. As in our goal, we can see that all mobile sensors represented as green ' $x$ ' are covered by at least one drone while a path to the base station exists. If the drones 4 and 2 had not changed positions from time step 4 to 5 , they would still satisfy the constraints, but they exchanged positions traveling 25 meters in 2 seconds, which means they traveled at $12.5 \mathrm{~m} / \mathrm{s}$ consuming less energy than they would have done if they stayed hovering as we saw in Figure 2.

The same happens between drones 3 and 5. Drones 5 and 4 were 50 meters apart at time step 4 , so if they exchanged positions they would need to travel at $25 \mathrm{~m} / \mathrm{s}$ which would consume more energy than hovering. Therefore, increasing the time between time steps, tts, allows the drones to move at the optimal speed for longer distances which increases their mobility when minimizing their energy consumption.

In conclusion here, we have remarked that minimizing only the energy spent by the drones increases their mobility between time steps, while minimizing only the traveled distance encourages the drones to hover a long time in the same position. These two antagonistic objectives have to be included in an effective optimal model to derive solutions combining both objectives to maximize the FANET lifetime. In the following, we seek to look more carefully into the tradeoff between distance and energy.

\section{Trade-off analysis}

Here we analyze the trade-off between minimizing the total traveled distance or the total energy consumed. For this we use the objective function 10 with the number of deployment positions $|P|$ fixed as 9. All other parameters were used with their default values described before. We found the optimal solution for 9 different traces of the mobile sensors varying the value of $\alpha$ from 0 to 1 for each one of them. When minimizing the total traveled distance the optimal solution was on average $655.82 \mathrm{~m}$ with an average energy consumption of $18589.59 \mathrm{~J}$. On the other hand, when we minimize the energy consumption, the total traveled distance was on average $956.03 \mathrm{~m}$ while the minimal energy consumption average was $15872.28 \mathrm{~J}$. The trade-off between the two objectives can be seen in Figures 4 and 5.

In these scenarios that we tested, the mobility required from the drones to satisfy the constraints is very low. This means that several drones do not have to move between positions during the observation period. But the energy model adopted 

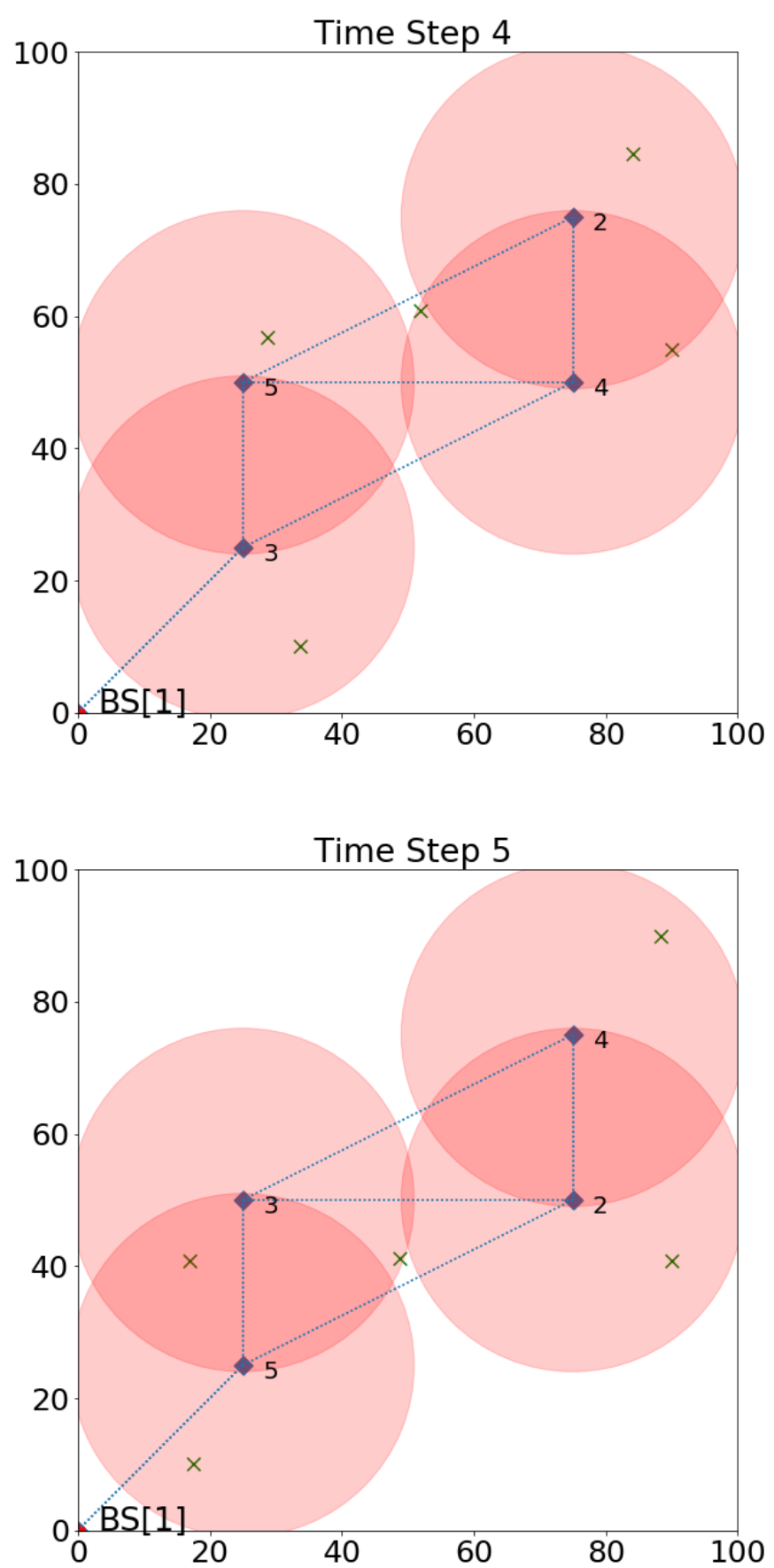

Fig. 3: Drones exchanging positions to minimize energy consumption.

gives us a lower energy consumption when the drones move at low speeds than the energy consumption when the drones hover a position. So as we minimize energy consumption, drones mobility increases.

But this doesn't mean that we need to choose between reducing the total traveled distance or energy consumption. As we can see in figure 6, when minimizing both the traveled distance and the energy consumption, we find intermediaries

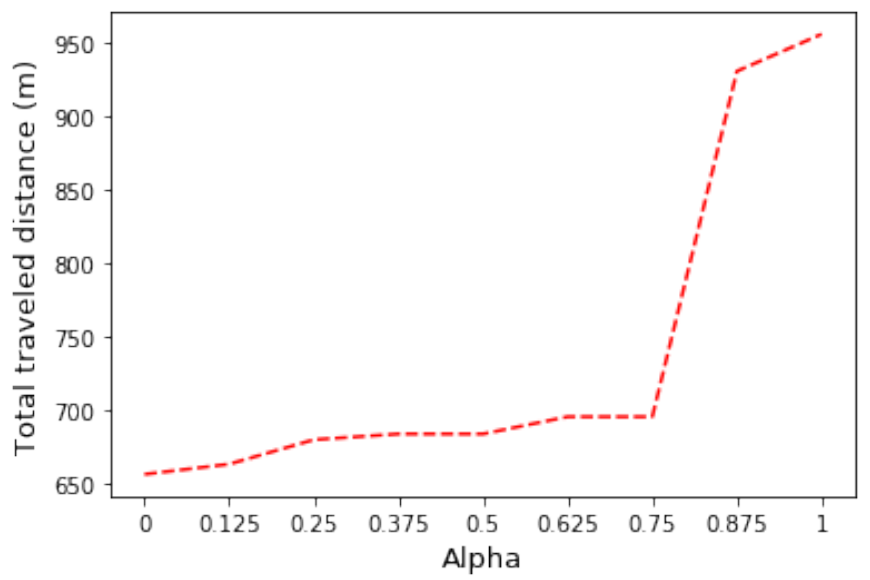

Fig. 4: Average of total traveled distance with objective function 10 as we vary alpha.

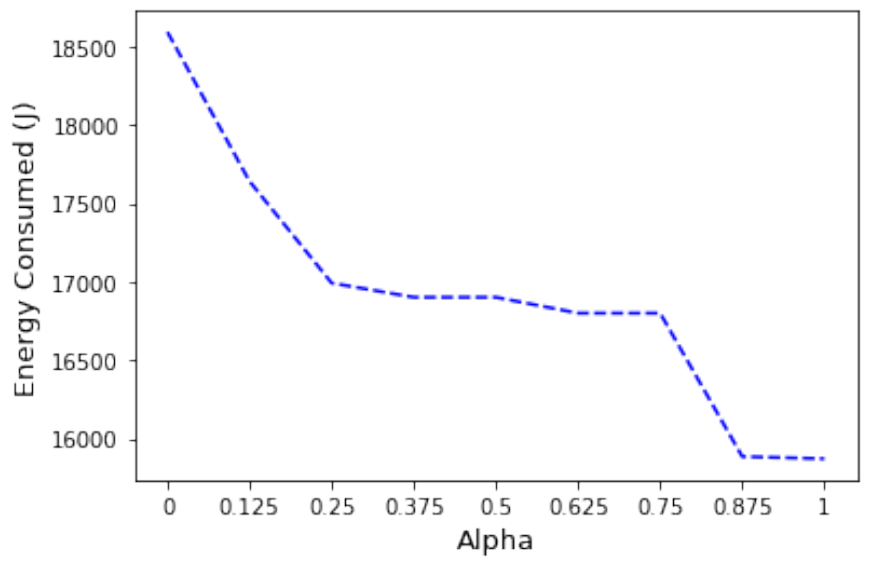

Fig. 5: Average of total energy consumed with objective function 10 as we vary alpha.

solutions that are good with values not far from the optimal ones for both the traveled distance and the energy consumed. For example, when $\alpha=0.5$ the solutions have on average a total traveled distance of 683.34 meters and consumed energy of 17432.84 Joules, which is a good compromise that fairly balance distance and energy.

\section{Resolution time}

We can see that CPLEX needs more time to find the optimal solutions for energy consumption than it needs to find the optimal solutions for the total traveled distance. This happens because to minimize the energy consumption the drones must have higher mobility as we can see in figure 4. As a consequence of the higher mobility, CPLEX has to analyze many more possibilities which results in higher time consumption to find the optimal solution. We can see this in figure 7, where we use the objective function 10 and vary the value of $\alpha$. When we minimize only the total traveled distance with $\alpha=0$ the average solution time is 54 seconds, but when 


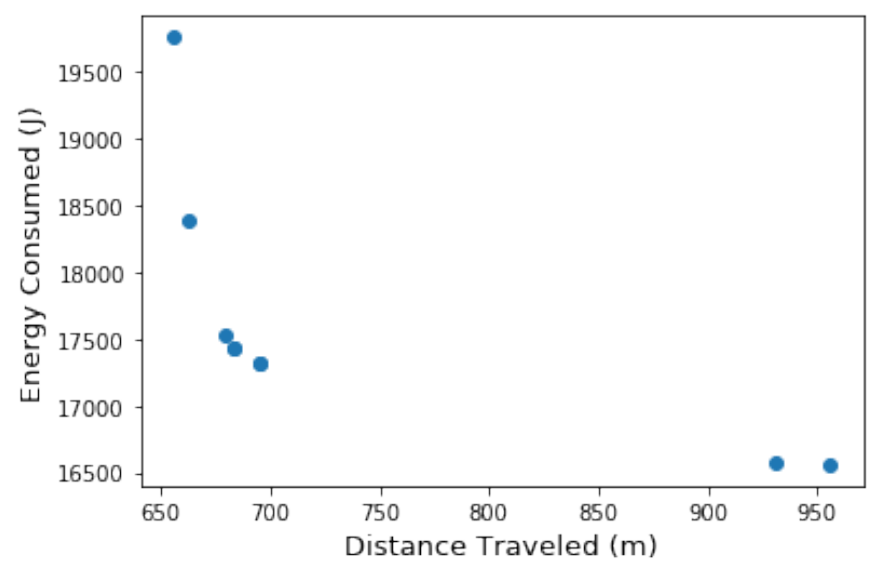

Fig. 6: The average energy consumption versus the total traveled distance with objective function 10 as we vary alpha.

we minimize only the energy consumption with $\alpha=1$ the average goes to 12.6 minutes.

These possibilities also increase with the number of drones, sensors, and positions, therefore all of these parameters also have a high impact on the resolution time. For example, in table I, we can see how quickly the average resolution time increases along with the deployment positions number going from 3.5 seconds with 9 deployment positions to 2.82 hours with 25 deployment positions. Note that with the same parameters, when we minimize the traveled distance with the objective function 8 , the average resolution time is 3.5 seconds and when using the objective function 10 with $\alpha=0$, the average is 54 seconds. In theory they are the same objective function, but in the implementation the objective function 10 has the energy terms multiplied by zero and they have this impact on the resolution time.

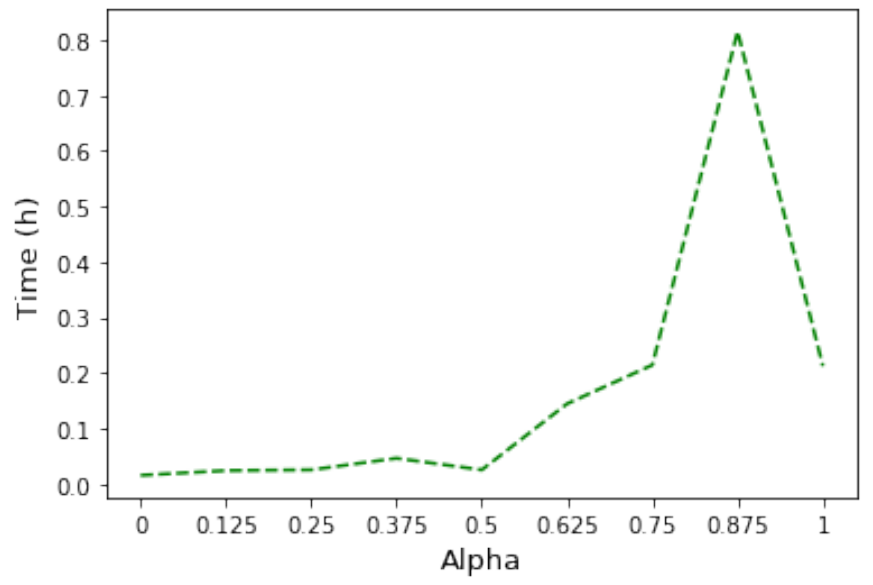

Fig. 7: Average of time to find solution with objective function 10 as we vary alpha with tts of 2 seconds.

Also, if we increase the time between time steps we increase the mobility of the drones because they can travel farther in the optimal speed. Therefore, increasing the $t t s$ also increases the time needed to find the optimal solutions as we can see comparing figures 8 and 7 .

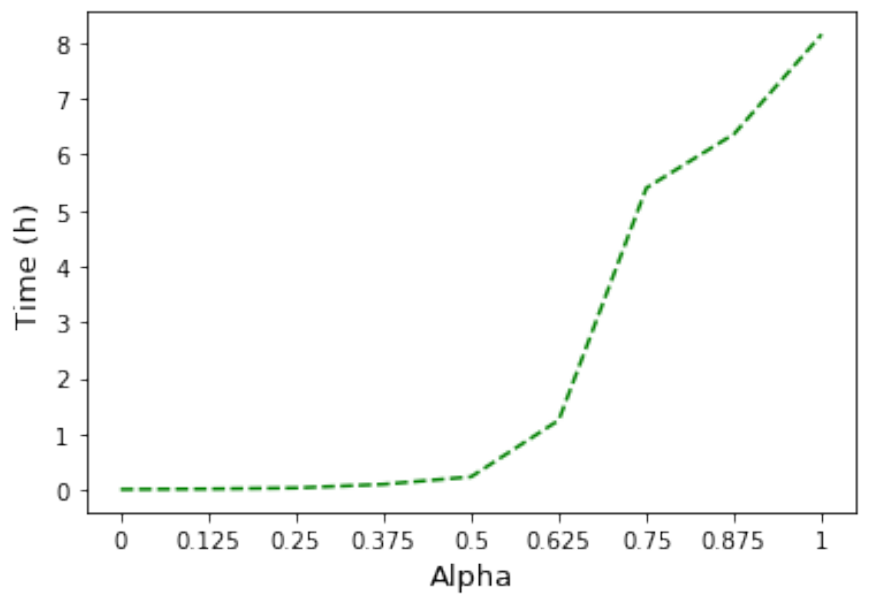

Fig. 8: Average of time to find solution with objective function 10 as we vary alpha with tts of 3 seconds.

\section{CONCLUSIONS}

In this paper we have presented an optimal model to obtain the trajectories of the drones for covering a set of mobile sensors and provide a backbone network connected to a base station at all times. With this model we can obtain the optimal trajectories that satisfy coverage and connectivity constraints and minimize the drones' total traveled distance or their energy consumption. In past optimization models, minimizing the drones' total traveled distance was equivalent to minimizing their energy consumption. But we used a more precise energy consumption model and showed that there is a significant trade-off between the distance traveled and the energy consumption. We also proposed an objective function to minimize the traveled distance and energy consumption simultaneously according to a given weight. With this objective function we can obtain fair solutions such that on average, both the total traveled distance and the energy consumed are close to their optimal values.

But this model requires a lot of time and memory to find the optimal solution so it cannot be applied for big instances of the problem where we consider many possible deployment positions, drones, mobile sensors, and a long observation period. Also, we need to know the trajectory of all mobile sensors beforehand to use this model which limits the scenarios where it can be applied.

Nonetheless, this model is an important first step that can be used to evaluate future heuristics by analyzing how far they are from optimal solutions and how much faster their solutions can be obtained. As our future work, we intend to improve the resolution time of our model by applying methods such as column generation so we can use it in bigger instances of the problem. 


\section{ACKNOWLEDGMENTS}

The authors want to thank Bai Xin for his fruitful comments about this work.

This work has been partially supported by the Capes Foundation within the Ministry of Education, Brazil (grant n. BEX 88887.361441/2019-00).

\section{REFERENCES}

[1] V. Dyo, S. A. Ellwood, D. W. Macdonald, A. Markham, N. Trigoni, R. Wohlers, C. Mascolo, B. Pásztor, S. Scellato, and K. Yousef, "Wildsensing: Design and deployment of a sustainable sensor network for wildlife monitoring," ACM Transactions on Sensor Networks (TOSN), vol. 8, no. 4, pp. 1-33, 2012.

[2] D. Zorbas and B. O'Flynn, "A network architecture for high volume data collection in agricultural applications," 052019.

[3] E. Yanmaz, M. Quaritsch, S. Yahyanejad, B. Rinner, H. Hellwagner, and C. Bettstetter, "Communication and coordination for drone networks," in Ad Hoc Networks. Springer, 2017, pp. 79-91.

[4] G. Guido, V. Gallelli, D. Rogano, and A. Vitale, "Evaluating the accuracy of vehicle tracking data obtained from unmanned aerial vehicles," International journal of transportation science and technology, vol. 5, no. 3, pp. 136-151, 2016.

[5] Y. Busnel, C. Caillouet, and D. Coudert, "Self-organized UAV-based Supervision and Connectivity: Challenges and Opportunities," in NCA 2019 - 18th IEEE International Symposium on Network Computing and Applications. Cambridge, United States: IEEE, Sep. 2019, pp. 1-5. [Online]. Available: https://hal.inria.fr/hal-02267396

[6] I. Bekmezci, O. K. Sahingoz, and Ş. Temel, "Flying ad-hoc networks (fanets): A survey," Ad Hoc Networks, vol. 11, no. 3, pp. 1254-1270, 2013.

[7] C. Caillouet, F. Giroire, and T. Razafindralambo, "Efficient data collection and tracking with flying drones," Ad Hoc Networks, vol. 89, pp. 35-46, 2019.

[8] D. Zorbas, L. D. P. Pugliese, T. Razafindralambo, and F. Guerriero, "Optimal drone placement and cost-efficient target coverage," Journal of Network and Computer Applications, vol. 75, pp. 16-31, 2016.

[9] L. D. P. Pugliese, F. Guerriero, D. Zorbas, and T. Razafindralambo, "Modelling the mobile target covering problem using flying drones," Optimization Letters, vol. 10, no. 5, pp. 1021-1052, 2016.

[10] L. Zhen, M. Li, G. Laporte, and W. Wang, "A vehicle routing problem arising in unmanned aerial monitoring," Computers Operations Research, vol. 105, pp. 1 - 11, 2019. [Online]. Available: http://www.sciencedirect.com/science/article/pii/S0305054819300012

[11] C. Caillouet and T. Razafindralambo, "Efficient Deployment of Connected Unmanned Aerial Vehicles for Optimal Target Coverage," in GIIS 2017 - Global Information Infrastructure and Networking Symposium, IEEE, Ed., Saint-Pierre, France, Oct. 2017. [Online]. Available: https://hal.inria.fr/hal-01575618

[12] A. Thibbotuwawa, P. Nielsen, B. Zbigniew, and G. Bocewicz, "Energy consumption in unmanned aerial vehicles: a review of energy consumption models and their relation to the uav routing," in International Conference on Information Systems Architecture and Technology. Springer, 2018, pp. 173-184.

[13] Y. Zeng, J. Xu, and R. Zhang, "Energy minimization for wireless communication with rotary-wing uav," IEEE Transactions on Wireless Communications, vol. 18, no. 4, pp. 2329-2345, 2019. 